\title{
Haptic identification of curved surfaces
}

\author{
ASTRID M. L. KAPPERS, JAN J. KOENDERINK, and INGE LICHTENEGGER \\ Utrecht Biophysics Research Institute, Utrecht, The Netherlands
}

\begin{abstract}
In two experiments, the active haptic identification of three-dimensional mathematically welldefined objects is investigated. The objects, quadric surfaces, are defined in terms of the shape index, a quantity describing the shape, and curvedness, a quantity describing overall curvature. Both shape index and curvedness are found to have a significant influence on haptic shape identification. Concave surfaces lead to a larger spread in responses than convex ones. Hyperbolic surfaces show a slight tendency to be identified with more difficulty than elliptic ones. Surfaces with a high curvedness are identified more easily than those with a low curvedness. Results from experiments with constant and with random curvedness are indistinguishable. It is concluded that shape index and curvedness are psychophysically not confounded.
\end{abstract}

Haptic sense is of major importance for the perception of the three-dimensional (3-D) shape of tangible objects. An observer will often actively explore an object by using a combination of optics and haptics. Although the importance of active haptic exploration was recognized already by Katz (1925), Révész (1938), and Gibson (1962), hardly any quantitative psychophysical data on haptic perception exist (see, e.g., Loomis \& Lederman, 1986). The aim of our research is to collect a coherent body of quantitative data on haptic shape perception of 3-D, roughly handsized objects by active exploration. This paper addresses the first step toward such a goal: haptic identification of curved surfaces by active exploration with only minor restrictions.

Few studies have come close to the objectives of our research. Goodwin, John, and Marceglia (1991) and Goodwin and Wheat (1992) have presented quantitative results of experiments in which the subjects had to discriminate spherically curved surfaces applied to their fingerpads. Because Goodwin and colleagues were interested in the capacity of human observers to discriminate and to judge the scale of objects of constant curvature by using only information from the cutaneous receptors, they avoided active exploration in their experiments. With some justice, they argue that the results of active exploration experiments are almost impossible to interpret in terms of the roles of cutaneous afferents, since it is complicated to separate the sensory and motor effects in such tasks. Notwithstanding such arguments, it is important and interesting to learn more about the human capabilities of actively discriminating or identifying 3-D objects.

The authors wish to acknowledge the indispensable help of $\mathrm{H}$. Kolijn in the process of manufacturing the shapes and setting up the haptics laboratory. They also want to thank C. J. M. Wijgerde for his kind help with the software for the milling machine and $A$. J. Noest for his valuable comments. Correspondence should be addressed to A. M. L. Kappers, Utrecht Biophysics Research Institute, Princetonplein 5, 3584 CC Utrecht, The Netherlands (e-mail: a.kappers@fys.ruu.nl).
Gibson (1963) used 10 sculptured objects of unfamiliar shape in a series of matching and discrimination experiments, concluding that an ordinary observer can easily distinguish such a set of objects. Klatzky, Lederman, and Metzger (1985) carried out an identification experiment with the use of one hundred familiar objects, which led to a similar conclusion-namely, that active haptic object recognition can be both rapid and accurate. However, owing to the lack of a mathematical description of the objects in the preceding two studies, little more has been learned than that such a task is feasible (though this is important in itself!). Burton, Turvey, and Solomon (1990) approach the active perception of shapes from a different angle. Instead of touching the objects, their subjects are only allowed to wield them with a handle while they perform matching or discrimination tasks. These experiments have yielded quantitative results on active haptic perception, but since the subjects have only indirect access to the object, this study only partially addresses the kind of research questions we have in mind. Gordon and Morison (1982) investigated the active haptic perception of curvature in a quantitative manner. In a series of experiments, they determined different kinds of curvature thresholds, in all cases by using stimuli smaller than the size of a finger. They concluded that the perception of curvature was most sensitive when scanning movements were small and did not involve movement of the arm. Roland and Mortensen (1987) investigated the active haptic discrimination of spheres, ellipsoids, and rectangular parallelepipeda of different sizes. They compared their results with data from a simulation model. Finally, Davidson (1972) investigated the relationship between active handling and veridical haptic curvature perception. Though his main interest concerned the different scanning techniques and the resulting performance of different subjectsin particular blind and sighted humans-his paper also contains quantitative data on the active perception of onedimensionally curved strips. He reports that for horizontally placed strips, subjects, both blind and sighted, make more errors in categorizing concave stimuli than convex 
ones. For vertical strips, no such difference is found. This study provided the background for our own experiments on the identification of surfaces curved in two directions.

In haptic experiments with 3-D mathematically welldefined objects, one has to deal with some major problems that are typically not encountered in visual psychophysics-namely, the production and subsequent storage of the stimuli. At our laboratory, we have at our disposal a computer-controlled milling machine on which over 150 shapes have been produced. Since at this moment they fill about $20 \mathrm{~m}$ of shelf space in two cupboards, we have not yet exceeded the logistical constraints yielded by a psychophysical experiment.

The surfaces used in the experiments are curved in two dimensions. Mathematically, those surfaces can be described as

$$
z(x, y)=\frac{1}{2}\left(k_{1} x^{2}+k_{2} y^{2}\right)
$$

where $k_{1}$ and $k_{2}$ are the two principal curvatures and the coordinates $(x, y, z)$ are measured along the axes of an orthonormal frame. By varying $k_{1}$, and $k_{2}$, one can obtain all kinds of convex or concave, elliptic or hyperbolic paraboloids of different sizes. In order to define concave and convex, we use the convention that the material of the object occupies $z<z(x, y)$. On smooth surfaces, one may approximate the surface in a sufficiently small neighborhood of any of its points with such quadric shapes. In this sense, our stimuli exhaust the possible local shapes.

For most psychophysical experiments, it is necessary to distinguish shape from size. Koenderink (1990) and Koenderink and van Doorn (1992) have proposed two quantities-namely, the shape index, $S$, as a measure of shape, and the curvedness, $C$, as a measure of size or overall curvature. Both curvedness and shape index have turned out to be very useful quantities in visual psychophysical experiments (de Vries, Kappers, \& Koenderink, 1991, 1993; Erens, Kappers, \& Koenderink, 1991, 1993; Hines \& Braunstein, 1993; van Damme \& van de Grind, 1991). $S$ and $C$ are defined as follows:

$$
S=-\frac{2}{\pi} \arctan \frac{k_{1}+k_{2}}{k_{1}-k_{2}} \quad k_{1} \geq k_{2}
$$

and

$$
C=\sqrt{\frac{k_{1}^{2}+k_{2}^{2}}{2}},
$$

where $S \in[-1,1]$ and $C \in[0, \infty) . S=-1$ corresponds with a concave symmetric elliptic paraboloid, whereas $S=1$ corresponds with the convex shape that exactly fits it (provided the curvednesses are identical). A hyperbolic paraboloid that exactly fits itself (after rotation) can be found at $S=0$, and concave and convex cylindrical paraboloids are found at $S=-0.5$ and $S=0.5$, respectively. For $C=0$, the surface is locally flat (then the shape is indefinite), and when $C$ approaches infinity, the surface

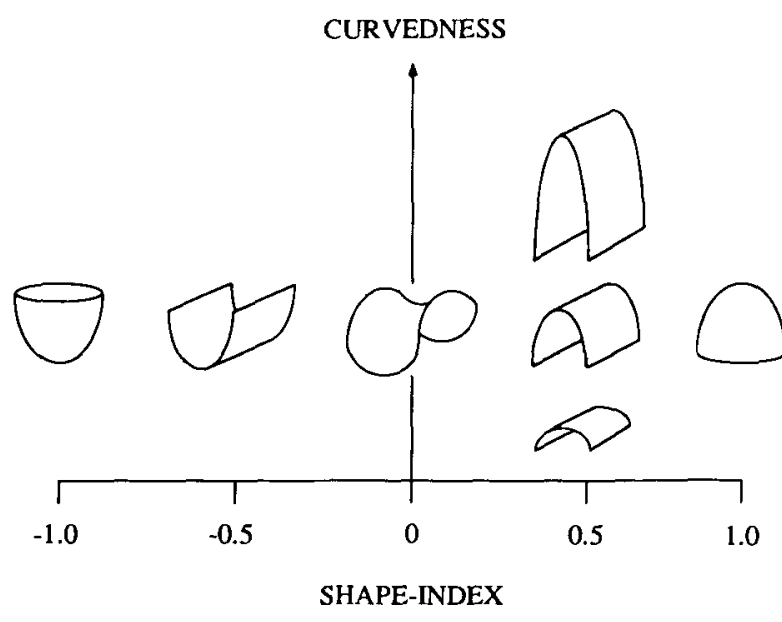

Figure 1. Schematic illustration of the shape index $(S)$-curvedness $(C)$ plane. $S$ varies between -1 (concave spherical paraboloid) and 1 (convex spherical paraboloid). $S=0$ corresponds with a hyperbolic paraboloid which exactly fits itself (after rotation), and -0.5 and 0.5 belong to a concave and convex cylinder, respectively. Between -1 and -0.5 and between 0.5 and 1 elliptical paraboloids are found, respectively concave and convex. Asymmetric saddles are located between -0.5 and 0.5 ( 0 excepted). $C$ varies from 0 (a flat surface) to infinity (an extremely curved surface). (Illustration drawn by $\mathbf{W}$. van Damme.)

is extremely curved. Both $C$ and $S$ are illustrated schematically in Figure 1.

In Figure 2, the relationship between $S$ and $C$ on the one hand and $k_{1}$ and $k_{2}$ on the other is illustrated graphically. For each combination of $k_{1}$ and $k_{2}$, the $S$ and $C$ values can be determined with Equations 2 and 3. Fixed ratios of $k_{1}$ and $k_{2}$ (on imaginary rays from the origin) correspond with fixed shape indices and thus constant shapes, whereas the curvedness can vary over the whole range $[0, \infty)$. For combinations of $k_{1}$ and $k_{2}$ which lie on circles around the origin, the curvedness is fixed and the shape index varies from $1\left(k_{1}=k_{2}\right)$ to $-1\left(k_{1}=-k_{2}\right)$. In the figure, only positions for which $k_{1} \geq k_{2}$ holds are marked; in the other half of the figure, shapes with $k_{1} \leq$ $k_{2}$ can be found. Because the choice of which principal curvature is termed $k_{1}$ and which is termed $k_{2}$ is arbitrary, both halves contain the complete set of second-order surfaces. If the principal curvatures are normally distributed random variables, all directions occur with equal probability. Since $S$ is just the angular orientation of the rays, it is uniformly distributed. Thus, the scale is geometrically uniform.

It should be noted that both $S$ and $C$ are locally defined quantities and thus that they typically vary over the surface. However, for experimental purposes, it is possible to construct surfaces on which $S$ and $C$ remain approximately constant. In such cases, $C$ is approximately equivalent to the "surface bending energy" used by other authors (e.g., Harris, 1987; Roland \& Mortensen, 1987; van Vliet \& Verbeek, 1993). 


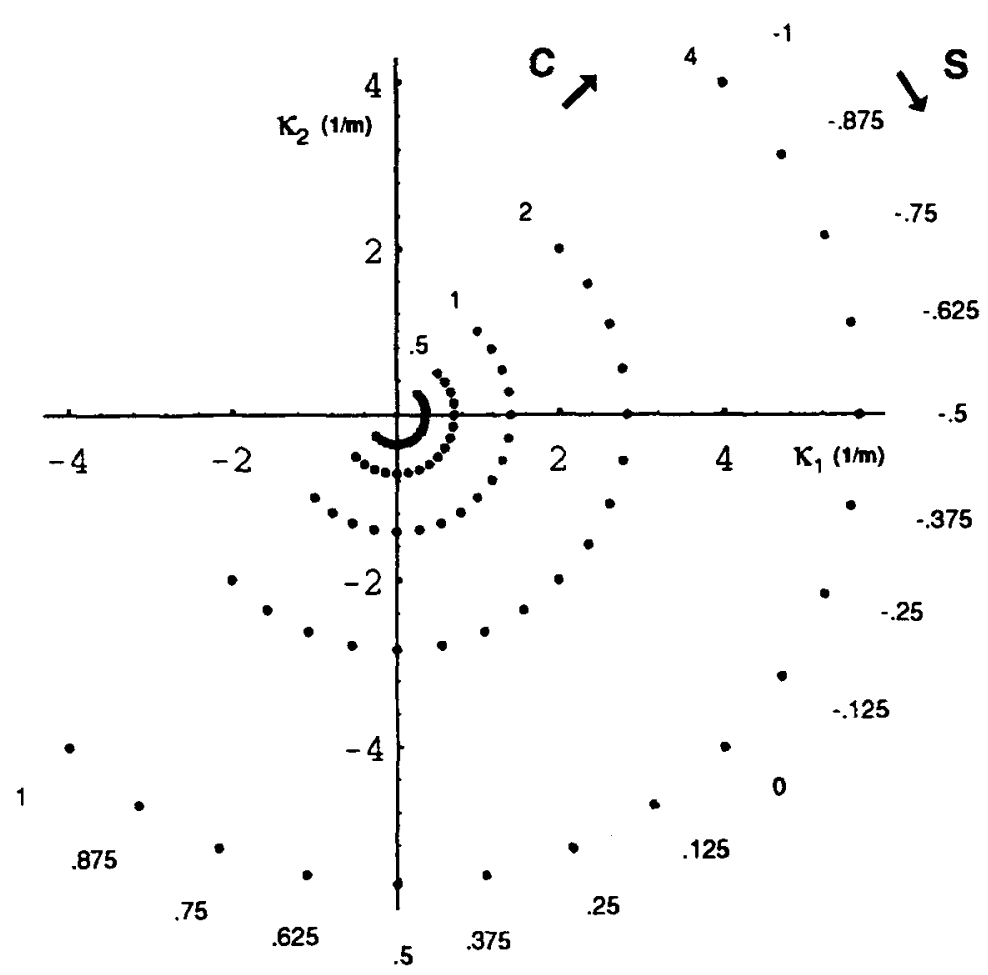

Figure 2. Ilustration of how $S$ and $C$ are related to $k_{1}$ and $k_{2}$. Shapes with equal curvedness $C$ lie on circles around the origin in the $k_{1}-k_{2}$ plane. Along such a circle the shape index $S$ varies from $1\left(k_{1}=k_{2}\right)$ to $-1\left(k_{1}=k_{2}\right)$. Only half of the circle is necessary to represent all different second-order shapes; the other half contains an identical set of shapes. The positions marked in the figure represent the shapes actually used in our experiments. The curvedness value of the inner circle is $0.25 / \mathrm{m}$.

\section{EXPERIMENT 1 \\ Identification of Surfaces With Constant Curvedness}

The purpose of the present experiments was to determine the extent to which subjects could haptically identify the shape of curved objects. From visual experiments, we know that human observers are quite able to use the shape-index scale (de Vries et al., 1991, 1993; Erens et al., 1991, 1993; van Damme \& van de Grind, 1991).

In the first experiment, the task of the subjects was to identify surfaces that differed only in shape index. To avoid a possible confounding effect of curvedness, we kept $C$ constant throughout a series. We hoped to answer two questions with this experiment: First, how would the performance of the subjects depend on curvedness, and second, how would the performance depend on shape index? The latter question addressed possible differences in identifying concave and convex, as well as elliptic and hyperbolic, surfaces. Although the $S$ scale is uniform geometrically, it need not be so perceptually.

\section{Method}

Stimulus. The material of the stimuli consisted of polyurethane foam impregnated with synthetic resin. The mechanical structure of this composite is comparable with beechwood. The stimuli are manufactured out of circular cylindrical blocks on a computercontrolled milling machine, which takes several hours per shape (the step size of the milling machine is $0.4 \mathrm{~mm}$; the error in the height is about $0.01 \mathrm{~mm}$ ). The bottom of the stimuli was flat and always rested on the table; the top was the smooth, curved surface. The diameter of all stimuli was $20 \mathrm{~cm}$; the height ranged between 3 and $8 \mathrm{~cm}$. An example of one of our stimuli, a hyperbolic paraboloid of shape index 0 (symmetric saddle) and curvedness $4 / \mathrm{m}$, is shown in Figure 3. In the bottom panel, the stimulus is shown with the hand of one of our subjects.

The stimuli had one of 17 shape-index values located at equal distances on the $S$ scale $(-1,-0.875,-0.75, \ldots 0.75,0.875$, 1). The sample density of the $S$ scale is a compromise between the desire to cover the scale with high resolution and the limitations on storage space and handling time. The curvedness was $0.25,0.5$, 1,2 , or $4 / \mathrm{m}$, thus spanning a range of spheres with radius $4-0.25 \mathrm{~m}$. Figure 2 illustrates where our stimuli lie in the $k_{1}-k_{2}$ plane.

As mentioned above, both the shape index and the curvedness vary somewhat over the shape; the values given hold for the center of tlte stimulus. However, since the curvedness is rather small, those variations are also relatively small. For shapes with $C=0.25 / \mathrm{m}$, both $S$ and $C$ vary less than $0.1 \%$. For shapes with $C=4 / \mathrm{m}, S$ varies less than $5 \%$; but for some shapes, $C$ varies as much as $35 \%$. The latter variation may seem unacceptably high, but those values only occur for certain shapes (the cylinder-like shapes) and only at the far boundaries. However, the variations are less than the interstimulus distance (both in $S$ and in $C$ ), and subjects are not asked to judge the curvedness.

Apparatus. The experiments were conducted in a small room specially equipped for our haptic studies. The subjects were seated in an armless chair at a table. The experimenter and the two cupboards with shapes were masked from the subject by a curtain hung halfway over the table. The subjects put their hands under the curtain 

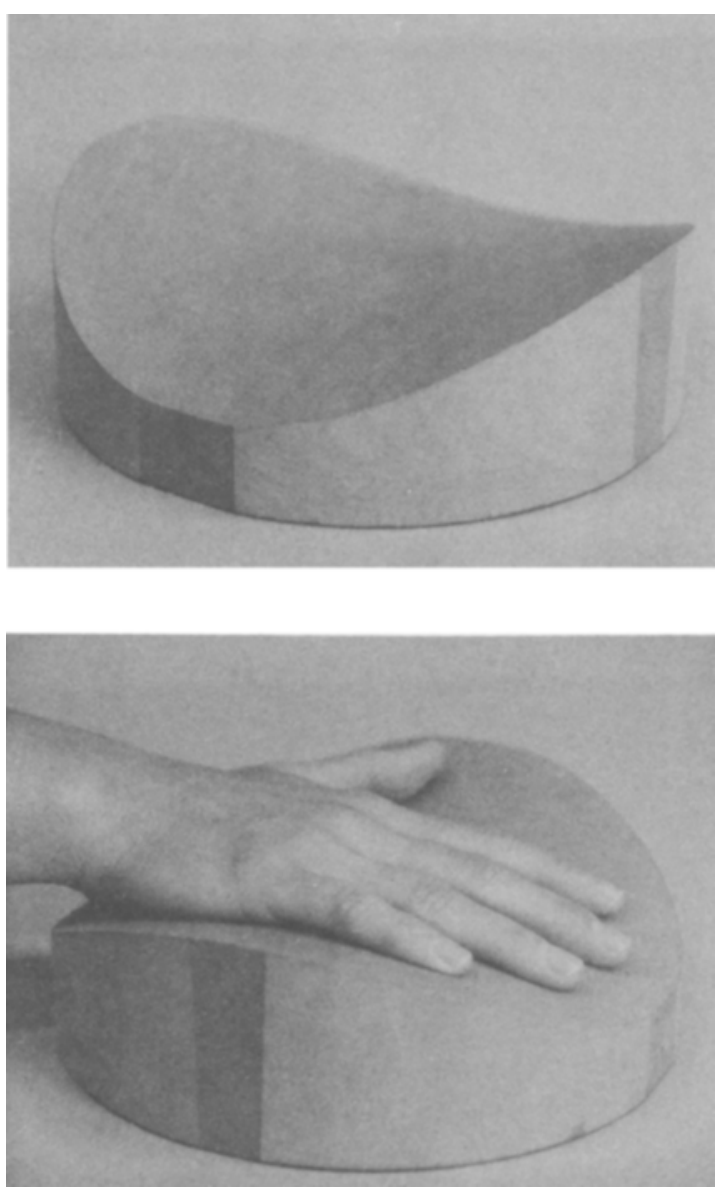

Figure 3. One of the stimuli: (top) the surface has a curvedness of $4 / \mathrm{m}$ and the shape index is 0 ; (bottom) same surface, with the hand of one of our subjects.

in order to touch the shapes, which thus remained unseen. It was possible to let 2 subjects perform the experiment at the same time.

Subjects. Three female and 2 male subjects participated in the experiment on a volunteer basis. None of the subjects reported any haptic deficiencies. The male observers performed only pilot experiments, whereas the female subjects participated in all series. The female observers (among them 2 of the authors) and 1 of the male observers were familiar with the subject of investigation. Subjects A.K., I.L., and S.P. also acted as experimenters and participated in other haptic experiments. The subjects used their dominant hand, which was in all cases the right one. Before the experimental sessions started, subjects were familiarized visually as well as haptically with the shape-index scale. This took little time, ${ }^{1}$ for there are several easily recognizable landmarks along the shapeindex scale (spherical paraboloids, 1 and -1 ; cylindrical paraboloids, . .5 and -.5 ; symmetric saddle, 0 ).

Procedure. The experimenter put a shape (invisible to the subject) with random orientation on the table and the subject was instructed to explore the shape in any way that the subject desired, provided that the bottom of the shape remained on the table and the contours were not systematically scanned. The subject was allowed only to touch the curved upper surface with one and the same hand; the other hand could be employed in rotating or fixing the shape. Exploration time was unlimited and varied widely with curvedness, shape index, and subject. The task of the subject was to identify the shape index of the object. The subjects noted their answers down in terms of the shape index on a special response list. The subjects were free to report any shape-index value between -1 and 1 , but in practice all but 1 of the subjects used only the values that were actually used in the experiment.

During each experimental session the curvedness was kept constant and the 17 different shapes were presented five times in random order. The time needed to complete a session of 85 identifications varied between .5 and $1.5 \mathrm{~h}$. For each of the five curvednesses, three sessions were run on different days. The only feedback given to a subject after each session was a scatterplot of the results. Feedback after each stimulus was considered to be undesirable because it might lead subjects to learn to identify the shapes on different (i.e., unwanted) grounds, such as small, unrecorded differences in height or texture.

\section{Results}

In Figure 4, representative results of 1 subject are presented as scatterplots. The data shown consist of a cumulation of the three experimental sessions with the same curvedness. The horizontal axis shows the shape index of the surface given to the subjects; the vertical axis shows the subject's response. The three scatterplots show results for three different curvednesses-the highest, the middle, and the lowest value used in the experiment (panel a, $C=$ $4 / \mathrm{m}$; panel b, $C=1 / \mathrm{m}$; panel c, $C=0.25 / \mathrm{m}$ ). Correct responses lie on the diagonal.

A number of observations can be made. First, the subjects' performance clearly depends on curvedness. A lower value of the curvedness results in a more scattered response diagram (compare Figures $4 a$ and $4 c$ ). Second, subjects confuse shapes most often with shapes having neighboring shape-index values, which indicates that the subjects are indeed able to use the $S$ scale.

A few interesting exceptions are to be found in Figure $4 c$, the condition with the lowest curvedness. The spread in answers is rather large; a number of outliers is immediately apparent. In some extreme cases the response is much closer to the opposite of the actual value than to the value itself; see, for example, data points $0.625,-0.625$ and $-0.625,0.625$. In those cases, the subject apparently still had some idea of the shape but evidently could not distinguish convex from concave (a consistent confusion of convex and concave would give rise to responses clustered around the other diagonal). These opposite responses do not occur often enough to justify the conclusion that at this curvedness subjects lost their ability to determine whether a surface was concave or convex. Experiments with an even smaller curvedness (which are not feasible at this moment, owing to a lack of stimuli) will probably not decide the issue, because the most obvious outcome will be an almost completely filled scatter diagram.

Although Figure 4 gives a good overview of the scatter in the responses of the subject, because of the overlap of responses it contains insufficient information to give access to a more concrete measure of spread such as the standard deviation. Therefore, in Figure 5, standard devi- 

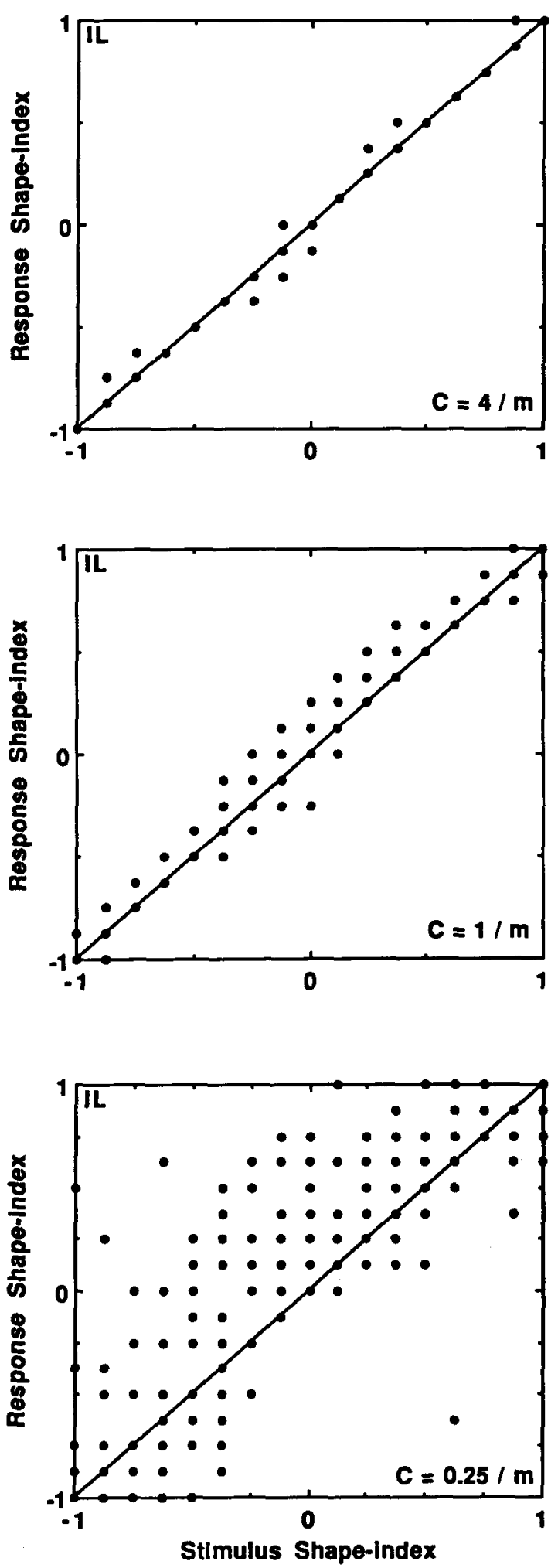

Figure 4. Results from 1 subject in the identification experiment with constant curvedness are shown for three different curvednesses. The horizontal axis denotes the stimulus shape index; the vertical axis the subject's response. Each shape index has been presented 15 times. Correct answers lie on the diagonal. (a) $C=4 / \mathrm{m}$, (b) $C=$ $1 / \mathrm{m}$, and (c) $C=0.25 / \mathrm{m}$. ations are given as a function of shape index for the five different values of the curvedness. Representative results of 2 subjects are shown. A full analysis of variance ${ }^{2}$ revealed that shape index, curvedness, and subject all had significant influence on performance. The only significant interaction was that of curvedness $X$ shape index. In Figure 4, the significant effect of curvedness can be observed easily: a higher curvedness, resulting in lower standard deviations [for I.L., $F(4,64)=56.9, p<.0001$; for A.K., $F(4,64)=63.2, p<.0001$; for S.P., $F(4,64)$ $=78.6, p<.0001]$. To investigate the kind of effect that the shape index had on performance, the shape-index range was divided into four regions: concave elliptic $(-1 \leq S<-0.5)$, concave hyperbolic $(-0.5<S<$ $0)$, convex hyperbolic $(0<S<0.5)$, and convex elliptic $(0.5<S \leq 1)$. In this analysis, ${ }^{3}$ shapes with $S=$ $-0.5, S=0$, and $S=0.5$ were left out of consideration, because they lay on the boundaries between two regions. Two of the subjects performed significantly less well (i.e., showed higher standard deviations) with concave shapes than with convex ones [for I.L., $F(1,54)=16.6$, $p<.0002$; for A.K., $F(1,54)=4.6, p<.04]$. For all subjects, there was a significant interaction between curvedness and "concaveness" [for I.L., $F(4,54)=6.3, p<$ .0003 ; for A.K., $F(4,54)=9.7, p<.0001$; for S.P., $F(4,54)=5.9, p<.0005]$. Finally, 2 subjects scored significantly lower with hyperbolic shapes [for I.L., $F(1,54)$ $=4.1, p<.05$; for S.P., $F(1,54)=7.5, p<.009]$.

\section{Explorative Strategies}

Although the scan strategies of the subjects were not recorded, it seems worthwhile to describe them briefly. This report is a combination of the notes of the experimenters and introspection of the subjects.

Subjects I.L. and S.P. used the right hand both to scan the shape and to rotate it, whereas Subject A.K. used her right hand only for scanning and her left hand only for fixing and rotating the shape. All subjects except R.E. (who did only a pilot session) palpated the surface with the entire hand instead of using only the fingers. Although free to do otherwise, subjects always made scanning movements. No attempt was ever made to base responses on information provided by a static hand.

The first action of the subjects was to determine the directions of the principal axes and to rotate the shape in such a way that one of the axes became aligned with the direction of the fingers. Obviously, this step was rather difficult in case of the smallest curvedness. The next step was to compare the curvatures of the two perpendicular directions. In the neighborhood of the cylinderlike shapes sometimes a different strategy was used. After determination of the principal axes, subjects systematically scanned the axis with the smallest absolute value of curvature in order to decide whether this axis was straight, convex, or concave.

There existed, of course, differences between the subjects in scanning movements. Our observations, however, were not detailed and systematic enough to allow further description. 

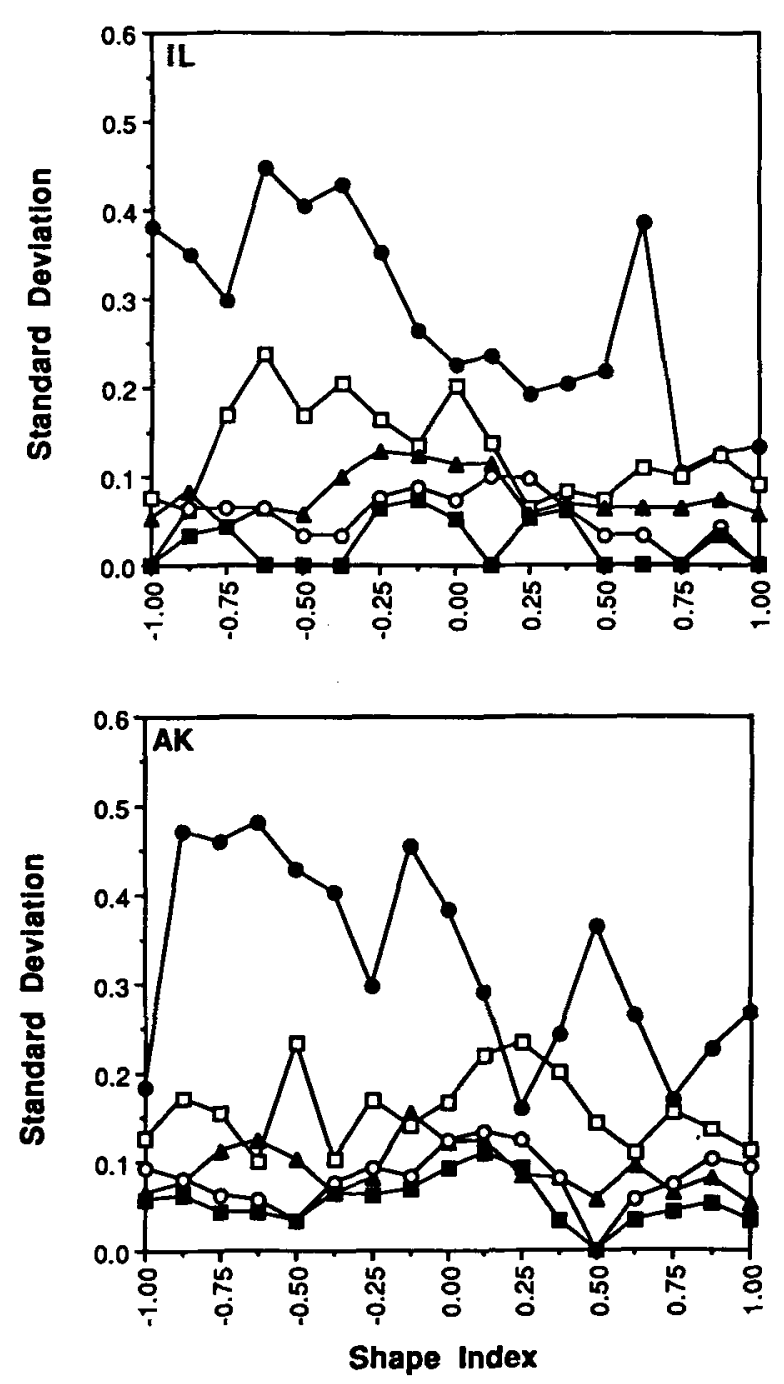

Figure 5. Identification experiment with constant curvedness. For 2 subjects, graphs of the standard deviation as a function of the shape index for the five different curvednesses used in the experiment. Filled circles: $C=0.25 / \mathrm{m}$. Open squares: $C=0.5 / \mathrm{m}$. Filled triangles: $C=$ $1 / \mathrm{m}$. Open circles: $C=2 / \mathrm{m}$. Filled squares: $C=4 / \mathrm{m}$.

\section{EXPERIMENT 2 \\ Identification of Surfaces With Random Curvedness}

In the first experiment, the curvedness was kept constant during each experimental session. As a consequence, the subjects could, in principle, have solved the task by only attending to one of the principal axes. The results would then reflect how well the subject was able to identify and scale this axis. If this strategy was indeed used, uncertainty in the curvedness should mar the results. If, on the other hand, subjects are able to systematically compare the two principal curvatures, the results will remain unaffected by uncertainty in $C$. Yet another possibility is that subjects used the second strategy but adapted some- how to the curvedness. Uncertainty about the curvedness will then again worsen the results.

The goal of the second experiment was to investigate the preceding question. Mathematically, $S$ and $C$ are orthogonal quantities. We hoped to determine whether or not the shape index and the curvedness were psychophysically confounded. Experiment 2 was similar to Experiment 1 , the difference being that now in each session both $S$ and $C$ were unknown to the subjects. Again, our main interest was directed toward the performance of the subjects as a function of shape index and of curvedness. The subjects had only to identify the shape index; the identification of curvedness was not tested.

\section{Method}

Stimulus and Apparatus. The stimuli and the experimental setup were identical to those in Experiment 1.

Subjects. Subjects A.K. and I.L. participated in Experiment 2. They again used their right hands. They acted in turn as observer and experimenter.

Procedure. In Experiment 2, both $S$ and $C$ were varied in each experimental session. A long random list was produced in which each of the 17 different shape indices paired with the five curvednesses occurred 15 times. Next, this list consisting of 1,275 items was split up into 15 sublists of 85 items. During each session, the shapes on one of the sublists were presented to the subject. In this way, all stimuli were tested just as many time as in the previous experiment. The task of the subject was again to identify the shape index of the stimulus and to write this value down on the response list. After each session, the subject was allowed to compare her response list to the list with the actual stimulus shapes. This was the only feedback provided during the experiment.

\section{Results}

In Figure 6, scatterplots are shown for the same subject as that in Figure 4, and it can be seen that the results are very similar. Again, the scatter reduces with an increase of curvedness. Moreover, for the smallest curvedness, a few opposite responses can be observed.

Figure 7 shows the standard deviation as a function of shape index for the 2 subjects. The high peaks for some of the convex elliptic shapes in the curve of $C=0.25 / \mathrm{m}$ in the graph of Subject A.K. are due to opposite responses, which were scored in just one session. The effect of curvedness on standard deviation was significant for both observers [for I.L., $F(4,64)=67.5, p<.0001$; for A.K., $F(4,64)=63.0, p<.0001]$. There was also a significant effect of shape index [for I.L., $F(16,64)=3.2, p<$ .0004 ; for A.K., $F(16,64)=1.9, p<.05]$. The effect of shape index was analyzed in the same manner as in Experiment 1. Again, subjects performed significantly better with convex shapes [for I.L., $F(1,54)=14.9, p \leq$ .0003 ; for A.K., $F(1,54)=5.8, p<.02]$. For Subject I.L., hyperbolic shapes led to lower performance $[F(1,54)=17.3, p<.0001]$, and there was a significant interaction of curvedness and "concaveness" $[F(4,54)=$ $4.4, p<.004]$.

In Figure 8, the average standard deviation is shown as a function of curvedness. In this way, the results of the two experimental conditions (constant and random curvedness) can be compared directly. This figure shows 


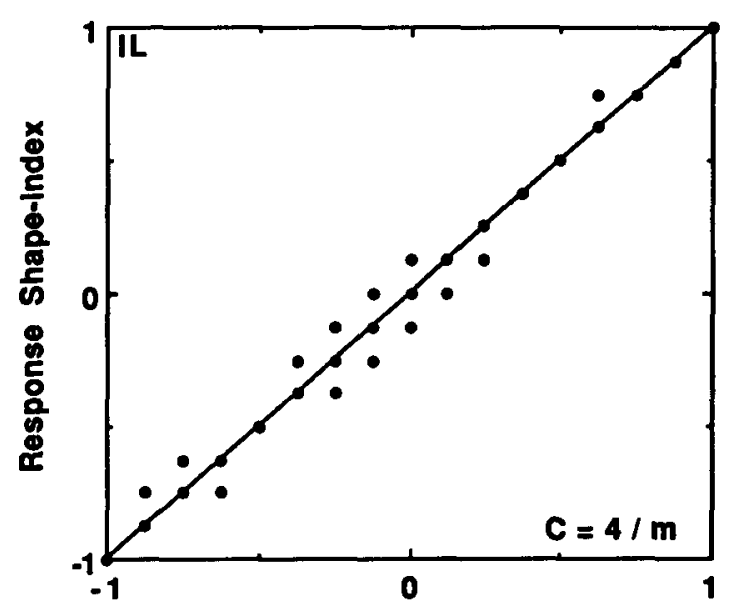

(a)

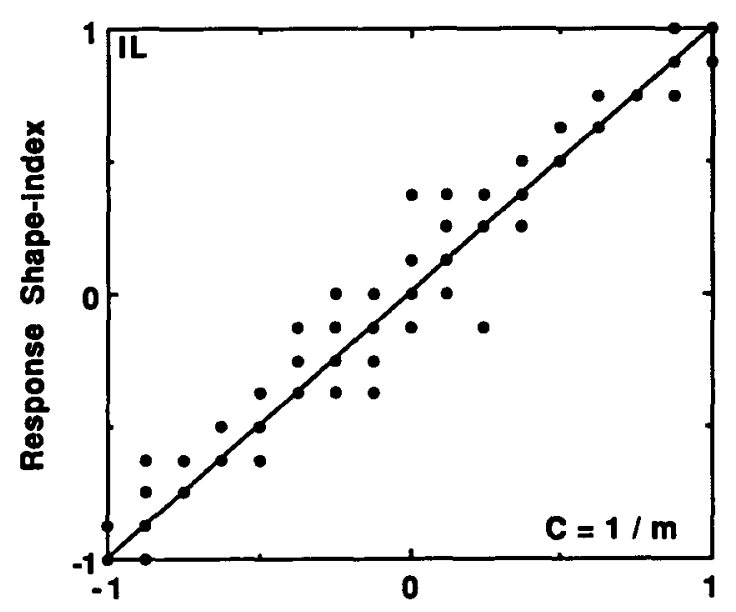

(b)

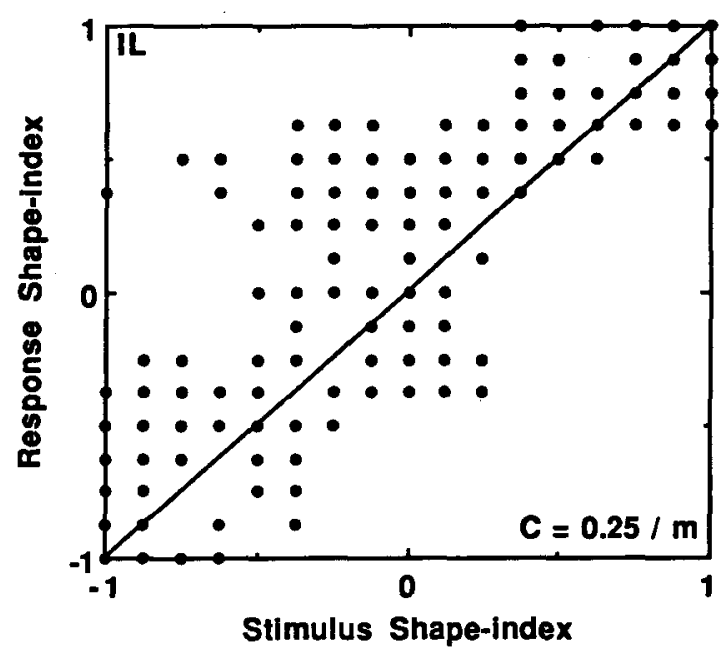

Figure 6. Results from 1 subject in the identification experiment with random curvedness are shown for three different curvednesses. The horizontal axis denotes the stimulus shape index; the vertical axis, the subject's response. Each shape index has been presented 15 times. Correct answers lie on the diagonal. (a) $C=4 / \mathrm{m}$, (b) $C=$ $1 / \mathrm{m}$, and (c) $C=0.25 / \mathrm{m}$. rather convincingly that there was no significant difference between the two conditions [for I.L., $F(1,64)=$ 0.04; for A.K., $F(1,64)=0.14]$.

On this double logarithmic scale, the relationship between the average standard deviation and the curvedness is linear, indicating a power function. For Subject I.L., the slope of this straight line is -0.84 ; for Subject A.K., it was -0.65 .

The strategies used by the 2 subjects were much the same as described previously. Subject I.L. reported that she was inclined more than before to compare the curvatures of the two perpendicular principal axes. Subject A.K. reported no difference at all.

\section{DISCUSSION}

Both experiments have shown a clear influence of curvedness on performance (see, e.g., Figure 8). This influence is not really surprising. Eventually, performance has to drop as curvedness decreases and curvatures become near or below threshold. Similarly, it might be expected that for high curvednesses subjects are able to distinguish 17 distinctive shapes equally spaced along the shape-index scale. The unknown factor is the exact nature of the relationship between performance and curvedness. This turned out to be a power function with exponents of -0.84 for Subject I.L. and -0.65 for A.K. A simple model, estimating the shape index from two perpendicular curvatures that are supposed to be the principal ones and assuming that only constant errors in the estimation of the height occur would predict an exponent of -1 .

The results also depend on shape index, with concave shapes yielding significantly higher standard deviations than convex ones. For Subject I.L., hyperbolic shapes also resulted in lower performance. In an attempt to explain the latter finding, an argument heard frequently is that in daily life human beings are much more often confronted with elliptic than with hyperbolic shapes. This, however, is a mistake, since every smooth object that contains concave patches also contains hyperbolic patches. Still, the fact that such a false idea exists indicates that hyperbolic shapes somehow lack familiarity. Similarly, convex shapes are considered to be more "natural" than concave shapes. We think that such suggestions can at most partially explain the results. Nevertheless, it is interesting that in visual experiments hyperbolic shapes are classified less accurately (de Vries et al., 1991, 1993; van Damme \& van de Grind, 1991).

The stimuli Davidson (1972) used in his experiment were 20-cm-long curved strips with an arc height of 2,4 , 6 , or $8 \mathrm{~mm}$. Those one-dimensionally curved stimuli fall well within the range of curvatures used in our experiments, so his results for vertical strips can be compared with those for our cylinders. In his experiments, the subjects' task was to categorize each stimulus as convex, concave, or straight, and his attention was focused on the veridical perception of curvature. One of his findings was that for vertical placement of the strip, the incorrect categorizations were distributed evenly over the three response 

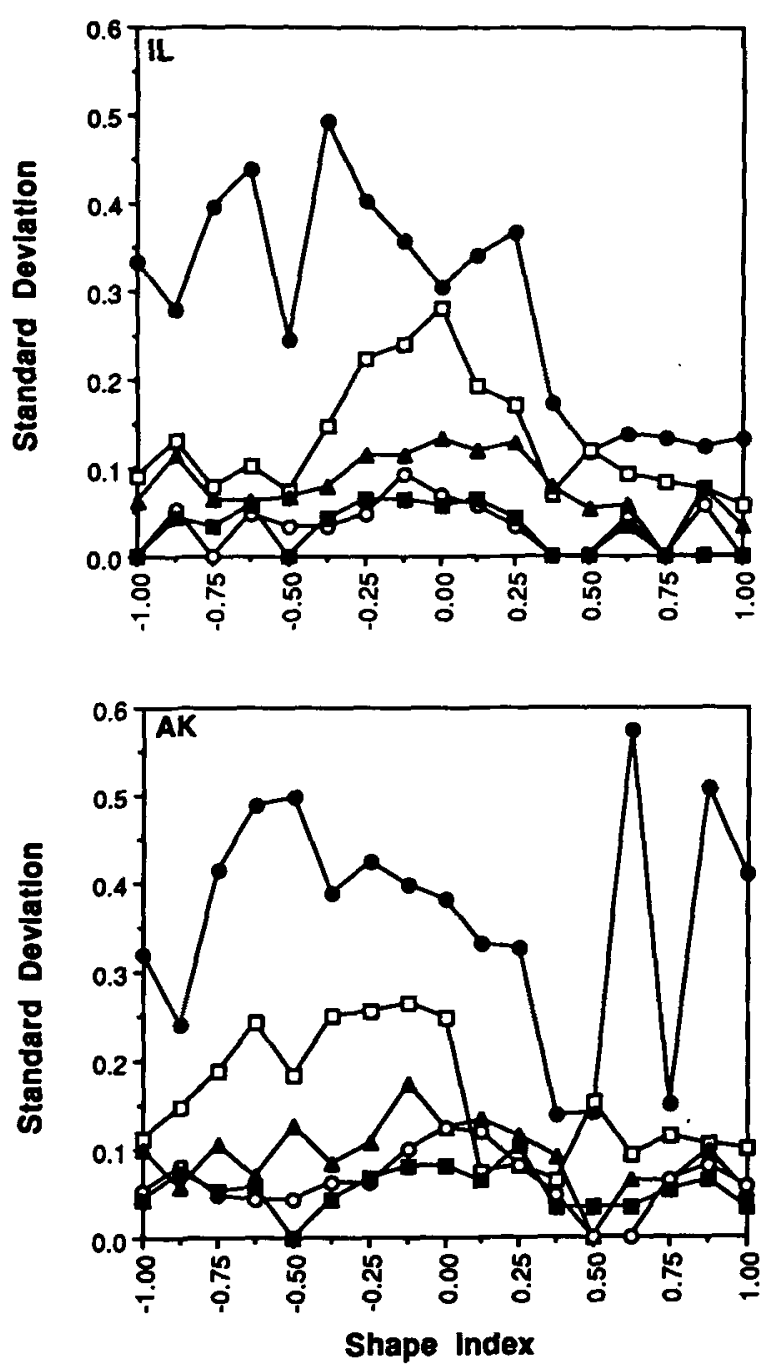

Figure 7. Identification experiment with random curvedness. For 2 subjects, graphs of the standard deviation as a function of the shape index for the five different curvednesses used in the experiment. Filled circles: $C=0.25 / \mathrm{m}$. Open squares: $C=0.5 / \mathrm{m}$. Filled triangles: $C=$ 1/m. Open circles: $C=2 / \mathrm{m}$. Filled squares: $C=4 / \mathrm{m}$.

classes. He reported that the standard deviation was larger in the concave (in his terminology, convex!) case than in the convex one, which agrees with our findings.

Klatzky and Lederman (e.g., Klatzky \& Lederman, 1987; Lederman \& Klatzky, 1987) distinguish a number of typical movement patterns observed during several very diverse tasks. For shape recognition, contour following with the fingers was the most frequently observed exploratory strategy. In our experiments, such movements were not allowed, but it remains to be seen whether they would lead to sufficient information about the exact shape of the surfaces. None of the exploratory procedures defined by Klatzky and Lederman can be used to describe the strategies used by our subjects. The pattern movements our subjects made most often can best be characterized as surface following, which is a scanning movement made with the full hand.
In our experiments, subjects need a strategy to solve the task. The most obvious strategy in the identification process involves the comparison of the curvatures of the two perpendicular principal axes (if they can be identified). For the hyperbolic shapes, one of the curvatures is positive (i.e., concave), and for the concave elliptic shapes, both curvatures are positive. Since subjects tend to solve the task by touching the surface with the entire hand, the attitude of their hand varies widely with the curvature (positive or negative) of the axis. Because the angle that the fingers can make with respect to the hand is restricted, the available range of attitudes connected with positive curvatures is much more limited than that with negative curvatures. It might well be possible that this factor contributes to the fact that the judgment of positive curvatures is less accurate. Obviously, further experiments are needed in order to investigate this possibility.

The two identification experiments (constant or random curvedness) yielded indistinguishable performance; their
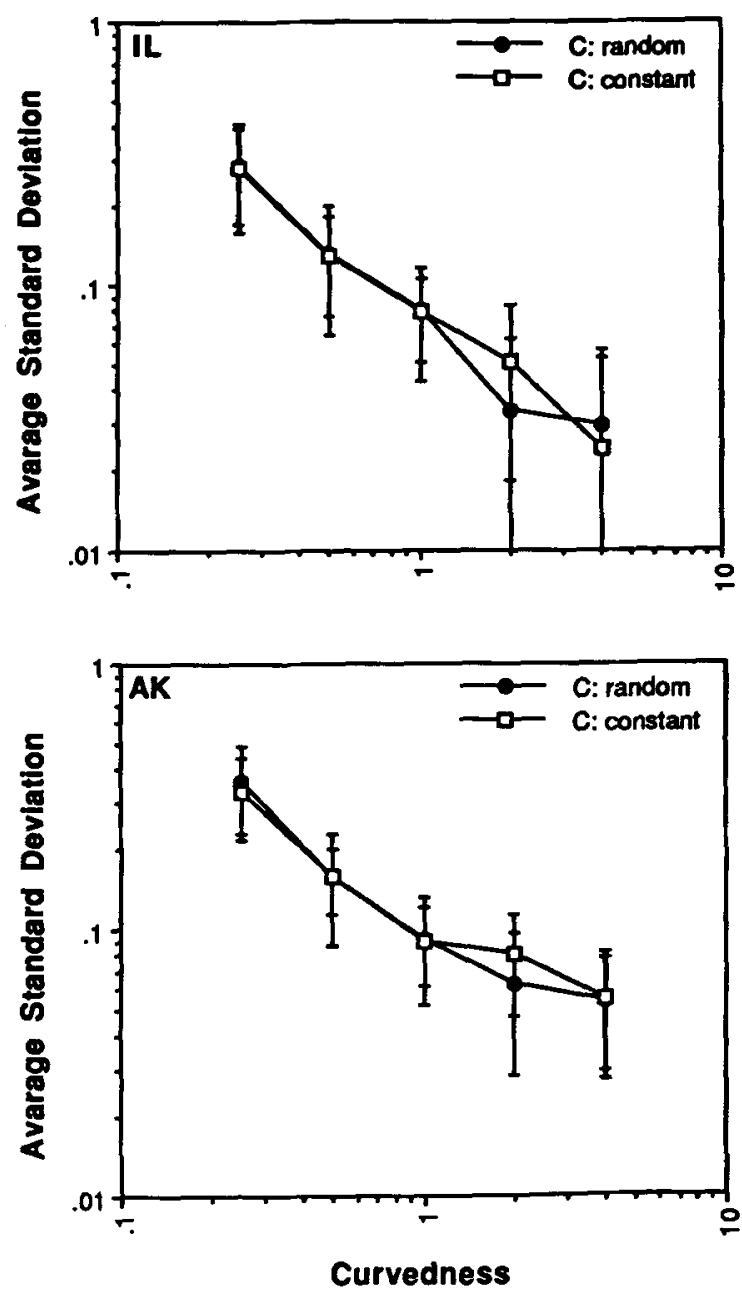

Figure 8: Standard deviation averaged over all shape indices as a function of the curvedness. Results of both the identification experiments with constant and random curvedness are shown for 2 subjects. 
results were identical. Thus, the fact that the curvedness is unknown did not influence the subjects' estimation of the shape index. This strongly supports evidence that $S$ and $C$ are psychologically not confounded.

Finally, the discrepancies between the average responses and the actual stimuli should be considered. The scatterplots of $C=4 / \mathrm{m}$ and $C=1 / \mathrm{m}$ (Figures $4 \mathrm{a}-4 \mathrm{~b}$, and Figures $6 a-6 b)$ show the responses as almost equally distributed on either side of the diagonal. On the other hand, Figures $4 c$ and $6 c$ seem to show a bias in the direction of convex responses. Although in some studies such a bias has been one of the major topics of concern (e.g., Davidson, 1972), we deliberately did not pay any attention to it for a number of reasons. In the first place, we do not consider our experiments suitable for investigating the nature and cause of such a bias. Feedback was given only after each session (for reasons mentioned earlier), and thus subjects were not given an adequate opportunity to align their internal scale with the actual shape-index scale. It was often noticed that in subsequent sessions the bias was directed the other way (i.e., if in the first experiment a subject tended to respond with an $S$ value higher than the actual value [positive bias], in the next experiment a tendency toward a negative bias was often found). Furthermore, the would-be bias varied from subject to subject, from curvedness to curvedness (for high curvednesses, a bias probably does not exist at all), and possibly also from shape index to shape index, in a capricious manner. Therefore, experiments more directly focused on determining a possible bias will be necessary before any conclusions concerning veridical shape index identification can be drawn.

\section{REFERENCES}

Burton, G., Turvey, M. T., Solomon, H. Y. (1990). Can shape be perceived by dynamic touch? Perception \& Psychophysics, 48 , 477-487.

DAvidson, P. W. (1972). Haptic judgments of curvature by blind and sighted humans. Journal of Experimental Psychology, 93, 43-55.

DE VRIES, S. C., Kappers, A. M. L., \& KoenderinK, J. J. (1991). 3D shape recognition with stereo. In P. J. Beek, R. J. Bootsma, \& P. C. W. van Wieringen (Eds.), Studies in perception and action (pp. 58-64). Amsterdam: Rodopi.

De Vries, S. C., Kappers, A. M. L., \& KoenderinK, J. J. (1993). Shape from stereo: A systematic approach using quadratic surfaces. Perception \& Psychophysics, 53, 71-80.

Erens, R. G. F., Kappers, A. M. L., \& KoenderinK, J. J. (1991). Limits on the perception of local shape from shading. In P. J. Beek, R. J. Bootsma, \& P. C. W. van Wieringen (Eds.), Studies in perception and action (pp. 65-71). Amsterdam: Rodopi.

Erens, R. G. F., Kappers, A. M. L., \& KoenderinK, J. J. (1993). Perception of local shape from shading. Perception \& Psychophysics, 54, 145-156.

Gimson, J. J. (1962). Observations on active touch. Psychological Review, 69, 477-491.

GiBson, J. J. (1963). The useful dimensions of sensitivity. American Psychologist, 18, 1-15. (Reprinted in E. Reed \& R. Jones [Eds.] [1982]. Reasons for realism [pp. 350-373]. Hillsdale, NJ: Erlbaum.)

Goodwin, A. W., John, K. T., Marceglia, A. H. (1991). Tactile discrimination of curvature by humans using only cutaneous information from the fingerpads. Experimental Brain Research, 86, 663-672.
Goodwin, A. W., \& WhEAT, H. E. (1992). Human tactile discrimination of curvature when contact area with the skin remains constant. Experimental Brain Research, 88, 447-450.

GoRDON, I. A., \& MoRison, V. (1982). The haptic perception of curvature. Perception \& Psychophysics, 31, 446-450.

HARRIS, J. G. (1987). A new approach to surface reconstruction: The coupled depth/slope model. In Proceedings of the First International Conference on Computer Vision (pp. 277-283). Washington, DC: IEEE Computer Society Press.

Hines, J., \& Braunstein, M. L. (1993). Effect of shape on surface detection from motion. Investigative Ophthalmology \& Visual Science, 34, 1083 .

KaTZ, D. (1925). Der Aufbau der Tastwelt. Leipzig: Verlag von Johann Ambrosius Barth.

Klatzky, R. L., \& Lederman, S. J. (1987). The intelligent hand. Psychology of Learning and Motivation, 21, 121-151.

KlatzKY, R. L., Lederman, S. J., \& Metzger, V. A. (1985). Identifying objects by touch: An "expert system." Perception \& Psychophysics, 37, 299-302.

Koenderink, J. J. (1990). Solid shape. Cambridge, MA: MIT Press.

Koenderink, J. J., \& van Doorn, A. J. (1992). Surface shape and curvature scales. Image \& Vision Computing, 10, 557-565.

LEDERMAN, S. J., \& KLATZKY, R. L. (1987). Hand movements: A window into haptic object recognition. Cognitive Psychology, 19, 342-368.

LoOMIS, J. M., \& LeDERMAN, S. J. (1986), Tactual perception. In K. R. Boff, L. Kaufman, \& J. P. Thomas (Eds.), Handbook of perception and human performance: Vol. II: Cognitive processes and performance (pp. 31.1-31.41). New York: Wiley.

RÉvész, G. (1938). Die Formenwelt des Tastsinnes. The Hague: Martinus Nijhof.

Roland, P. E., \& Mortensen, E. (1987). Somatosensory detection of microgeometry, macrogeometry and kinesthesia in man. Brain Research Reviews, 12, 1-42.

vAN DAMME, W., \& VAN DE GRIND, W. A. (1991). Identification of 3D shapes using motion parallax. In P. J. Beek, R. J. Bootsma, \& P. C. W. van Wieringen (Eds.), Studies in perception and action (pp. 72-75). Amsterdam: Rodopi.

van Vliet, L. J., \& Verbeek, P. W. (1993). Curvature and bending energy in digitized 2D and 3D images. In Proceedings of the 8th Scandinavian Conference on Image Analysis.

\section{NOTES}

1. In this respect, it is interesting to mention a small pilot experiment. Four naive subjects ( 2 male and 2 female) from outside the physics department were asked to order the 17 shapes with a curvedness of $4 / \mathrm{m}$ (F.K., H.K., and T.P.) or $2 / m$ (M.L.). Nothing about shapes, shape index, or curvedness, was explained to them, and it can safely be assumed that they were unfamiliar with the definition of second-order shapes. They were allowed to use both vision and touch. All 4 subjects ordered the shapes spontaneously according to the shape-index scale, with only a few (respectively, $0,1,1$, and 2 ) confusions of neighboring shapes.

2. The independent variables in this analysis were shape (17 levels) and curvedness ( 5 levels). The interaction of shape and curvedness was used as the error term.

3. The independent variables in this analysis were curvedness (five levels): hyperbolic versus elliptic, and concave versus convex. Three two-way interactions were examined: curvedness with hyperbolic versus elliptic, curvedness with concave versus convex, and hyperbolic versus elliptic with concave versus convex. The total degrees of freedom was based on 5 levels of curvedness and 14 levels of shape index. The 14 levels were divided among the four combinations of hyperbolic versus elliptic with concave versus convex and were the cell entries in the analysis of variance. The error term was based on these cell entries plus the three-way interaction.

(Manuscript received September 16, 1992; revision accepted for publication November 29,1993 .) 